\title{
Correlation between prostate-specific antigen and four renal function-associated parameters in different ethnicities in southwest China
}

\author{
RUICEN LI ${ }^{1}$, HUAIRONG TANG ${ }^{1}$, TING BAO ${ }^{1}$, HANWEI YANG ${ }^{1}$ and TAO JIN ${ }^{2}$ \\ ${ }^{1}$ Department of Health Management Center, West China Hospital, Sichuan University; \\ ${ }^{2}$ Department of Urology, Institute of Urology (Laboratory of Reconstructive Urology), West China Hospital, \\ Sichuan University, Chengdu, Sichuan 610041, P.R. China
}

Received January 19, 2020; Accepted July 14, 2020

DOI: $10.3892 /$ etm.2020.9272

\begin{abstract}
A limited number of studies have investigated the significance of cystatin $\mathrm{C}$, creatinine, uric acid and urea in prostate cancer. The present study aimed to explore the correlation between these molecules and total prostate-specific antigen (tPSA) levels using big data from patients of different Chinese ethnicities. Patients undergoing physical examination at the Medical Examination Center of West China Hospital (Chengdu, China) between January 2010 and May 2019 were retrospectively included. A $\chi^{2}$ test or Fisher's test and Kruskal-Wallis rank-sum test were used to compare categorical and continuous variables. Pearson's correlation coefficients (r) with $95 \%$ CI were also determined to assess the correlation between tPSA and cystatin, uric acid, creatinine and urea in the entire patient population and in different ethnicities. A total of 253,281 male patients were included and their mean age was $47.83 \pm 14.28$ years. The mean tPSA level of these patients was $1.15 \pm 1.88 \mathrm{ng} / \mathrm{ml}$. The mean levels of the renal function-associated parameters cystatin $\mathrm{C}$, uric acid, creatinine and urea were $0.91 \pm 0.19,388.02 \pm 77.37,83.94 \pm 55.89$ and $5.23 \pm 1.23 \mathrm{ng} / \mathrm{ml}$, respectively. In the total patient population, urea $(\mathrm{r}=0.0774$, $\mathrm{P}<0.0001)$, creatinine $(\mathrm{r}=0.0219, \mathrm{P}<0.0001)$ and cystatin $(\mathrm{r}=0.1513, \mathrm{P}<0.0001)$ were slightly positively correlated with tPSA, whereas uric acid was negatively correlated with tPSA $(\mathrm{r}=-0.0307, \mathrm{P}<0.0001)$. Subgroup analyses generally yielded consistent results; however, a stronger correlation was noted between cystatin $\mathrm{C}$ and tPSA for the Mongolian ethnicity $(r=0.6572, P<0.0001)$ and between creatinine and tPSA for the
\end{abstract}

Correspondence to: Professor Tao Jin, Department of Urology, Institute of Urology (Laboratory of Reconstructive Urology), West China Hospital, Sichuan University, 37 Guoxue Xiang, Chengdu, Sichuan 610041, P.R. China

E-mail: jintaouro@126.com

Key words: prostate-specific antigen, renal function-associated parameters, ethnicity, southwest China
Yi ethnicity $(\mathrm{r}=0.6125, \mathrm{P}<0.0001)$. In conclusion, the present study used data from a large population to reveal a generally significant and slightly positive correlation between tPSA and cystatin $\mathrm{C}$ levels among the 10 most common ethnicities in China. Subgroup analyses indicated that the tPSA level was moderately positively correlated with the creatinine level for the Mongolian and Yi ethnicities and the cystatin $\mathrm{C}$ level was moderately positively correlated with tPSA for the Mongolian ethnicity. Future studies are required to confirm and expand the present results.

\section{Introduction}

Prostate cancer ( $\mathrm{PCa}$ ) is the most frequently diagnosed urologic malignancy worldwide (1). Prostate-specific antigen (PSA) is the most widely employed biomarker for the screening for PCa since several landmark trials were performed (2-5). These studies demonstrated that increased PSA is associated with an increased risk of PCa, particularly for patients with total PSA (tPSA) of $>10 \mathrm{ng} / \mathrm{ml}$, whose risk of being diagnosed with PCa may be up to $50-70 \%$. Furthermore, PSA also has an important role in the selection of treatment strategies and prediction of biochemical recurrence $(6,7)$. Of note, age is thought to be strongly associated with the incidence of PCa and the European Association of Urology recommends that males aged $>40$ years undergo regular screening for PCa (8). However, $<60 \%$ of patients aged 40 years were estimated to have taken a PSA test (9).

Renal function-associated parameters, including uric acid, creatinine, urea and cystatin $\mathrm{C}$, are important factors assessed in routine blood tests. Studies have reported that these parameters may impact the metabolism and proliferation of cancer cells and predict the prognosis of patients treated for upper-tract urothelial carcinoma, hepatocellular carcinoma and colon cancer (10-14). However, a limited number of studies investigated the significance of these parameters in PCa and only one study investigated the correlation between uric acid and tPSA levels (15). Therefore, the present study aimed to explore the correlation between further renal function-associated parameters and tPSA levels using big data of patients from different Chinese ethnicities undergoing regular physical 
examination at the Medical Examination Center of the largest hospital in southwest China. The possible correlation between PSA and these parameters was assessed, which may help identify patients with an increased risk of PCa based on abnormal values determined in different departments.

\section{Patients and methods}

Patients. The present study was a cross-sectional study. Patients undergoing a general physical examination at the Medical Examination Center of West China Hospital (Chengdu, China) between January 2010 and May 2019 were retrospectively identified from the system and screened for inclusion. The patients were classified based on the 10 most common ethnicities in China, including the Han, Zhuang, Hui, Miao, Tujia, Mongolian, Tibetan, Yi, Manchu and Qiang ethnicities. Patient data, including age, tPSA and renal function-associated parameters (cystatin $\mathrm{C}$, uric acid, creatinine and urea), were recorded. The ethics committee of West China Hospital (Chengdu, China) approved the present study.

Statistical analysis. Mean values with standard deviations were calculated for the above variables for the entire cohort and subpopulations. The $\chi^{2}$ test or Fisher's test and the Kruskal-Wallis rank-sum test was used to compare categorical and continuous variables of different ethnicities. Pearson's correlation coefficients (r) with 95\% CI were also analyzed to assess the correlation between tPSA and cystatin, uric acid, creatinine and urea in the entire cohort and different ethnicities. Correlation coefficient values (r) were calculated to indicate a positive ( 0 to 1$)$ or negative ( -1 to 0$)$ correlation. The absolute value of correlation coefficient was defined as 0-0.39 (weak), 0.4-0.6 (moderate) and 0.7-1 (strong) to indicate the strength of correlation (16). $\mathrm{P}<0.05$ was considered to indicate a statistically significant difference. Statistical analyses were performed using Empowerstats software (X\&Y Solutions, Inc.).

\section{Results}

Baseline characteristics of included patients. The characteristics of patients undergoing physical examination between 2010 and 2019 at the Medical Examination Center of West China Hospital (Chengdu, China) are presented in Table I. A total of 259,146 male patients were included and the mean age was $47.83 \pm 14.28$ years. The mean tPSA level in these patients was $1.15 \pm 1.88 \mathrm{ng} / \mathrm{ml}$. Most of the patients (96.99\%) had tPSA levels of $<4 \mathrm{ng} / \mathrm{ml}$. Furthermore, $2.55 \%$ had tPSA levels of $4-10 \mathrm{ng} / \mathrm{ml}$ and $0.46 \%$ had tPSA levels $>10 \mathrm{ng} / \mathrm{ml}$. The mean levels of renal function-associated parameters were $0.91 \pm 0.19,388.02 \pm 77.37,83.94 \pm 55.89$ and $5.23 \pm 1.23 \mathrm{ng} / \mathrm{ml}$ for cystatin $\mathrm{C}$, uric acid, creatinine and urea, respectively. In total, 255,914 patients of the present study were of the Han ethnicity, representing $98.75 \%$ of the study population. In total, 180 (0.07\%), 611 (0.24\%), $203(0.08 \%), 502(0.19 \%), 139(0.05 \%)$, $495(0.19 \%), 152(0.06 \%), 460(0.18 \%)$ and $225(0.09 \%)$ of the patients represented the Zhuang, Hui, Miao, Tujia, Mongolian, Tibetan, Yi, Manchu and Qiang ethnicities, respectively. Significant differences were observed among the ethnicities in cystatin $\mathrm{C}(\mathrm{P}<0.001)$, uric acid $(\mathrm{P}<0.001)$, creatinine $(\mathrm{P}<0.001)$, urea $(\mathrm{P}<0.001)$ and tPSA $(\mathrm{P}=0.007$; Table II $)$. Furthermore, the distribution of patients with tPSA $<4,4-10$ and $>10 \mathrm{ng} / \mathrm{ml}$ was also significantly different among the different ethnicities $(\mathrm{P}=0.036)$.

Correlation of IPSA with cystatin C, creatinine, uric acid and urea in the total population and different Chinese ethnicities. In the total population, all the four parameters, urea $(\mathrm{r}=0.0774$, $\mathrm{P}<0.0001)$, creatinine $(\mathrm{r}=0.0218, \mathrm{P}<0.0001)$, uric acid $(\mathrm{r}=-0.0307, \mathrm{P}<0.0001)$ and cystatin $\mathrm{C}(\mathrm{r}=0.1513, \mathrm{P}<0.0001)$, were weakly positively correlated with tPSA (Fig. 1). In the subgroup analysis, urea was positively correlated with tPSA among the Mongolian ( $\mathrm{r}=0.4562, \mathrm{P}<0.0001)$, Tibetan $(\mathrm{r}=0.1623, \mathrm{P}=0.0003)$, $\mathrm{Yi}(\mathrm{r}=0.3331, \mathrm{P}<0.0001)$ and Manchu ethnicities $(r=0.1864, P=0.0001)$. Similarly, a moderate positive correlation was noted between creatinine and tPSA for the Mongolian $(r=0.4616, \mathrm{P}<0.0001)$ and $\mathrm{Yi}$ ethnicities $(\mathrm{r}=0.6125, \mathrm{P}<0.0001)$. In addition, cystatin $\mathrm{C}$ was positively correlated with tPSA among the Han $(r=0.1511, P<0.0001)$, Zhuang ( $\mathrm{r}=0.3374, \mathrm{P}<0.0001)$, Tujia $(\mathrm{r}=0.1800, \mathrm{P}=0.0001)$, Mongolian ( $\mathrm{r}=0.6572, \mathrm{P}<0.0001)$, $\mathrm{Yi}(\mathrm{r}=0.2032, \mathrm{P}=0.0301)$ and Manchu ethnicities $(r=0.2395, \mathrm{P}<0.0001)$. However, uric acid was weakly negatively correlated with tPSA among the Qiang ( $r=-0.2345, \mathrm{P}=0.0004)$, Hui $(\mathrm{r}=-0.1289, \mathrm{P}=0.0015)$ and Manchu ethnicities ( $r=-0.1797, \mathrm{P}<0.0001$; Fig. 1).

\section{Discussion}

In the present study, the correlation between tPSA levels and uric acid, creatinine, cystatin $C$ and urea levels was investigated in a large patient population. Overall, a generally significant and slightly positive correlation was noted between tPSA levels and cystatin C levels among the 10 most common Chinese ethnicities. A potential reason accounting for the correlation being present in the total population but moderate in certain subgroups may be that the majority of the total population belonged to the Han ethnicity and the correlation was weak among Han Chinese. Subgroup analyses indicated that tPSA levels were moderately positively correlated with creatinine levels in Mongolian and Yi ethnicities, and cystatin C levels were positively correlated in the Mongolian ethnicity.

Multiple studies have reported the role of the four renal function-associated parameters in different cancer types. Cystatin $\mathrm{C}$ is an inhibitor of cancer invasion and metastasis by inactivating cathepsin protease activity $(17,18)$, and a previous study by Wegiel et al (19) reported that cystatin expression was significantly reduced in PCa tissues compared with benign non-malignant tissues as determined by immunohistology analysis. However, the present study indicated that the correlation between cystatin levels and tPSA levels (potential risk of PCa) was weak among different Chinese ethnicities except Mongolians. A possible explanation for the moderate correlation in Mongolians is that the homogeneous population exhibits increased expression of cystatin $\mathrm{C}$ in patients with $\mathrm{PCa}$ and higher tPSA levels. Another study by Weinstein et al (20), reported that creatinine levels within the normal range were positively associated with $\mathrm{PCa}$, while Wang (21) also indicated that the sarcosine/creatinine ratio was significantly different in the PCa group compared with that in the benign prostate hyperplasia group. Consistently with this, the present study indicated that creatinine levels were positively correlated with 
Table I. Characteristics of patients of the present study ( $n=259$, 146).

\begin{tabular}{lcc}
\hline Characteristics & Value & $\begin{array}{c}\text { Normal range of } \\
\text { laboratory parameters }\end{array}$ \\
\hline Age $($ years $)$ & $47.83 \pm 14.28$ & $0.51-1.09$ \\
Cystatin C $(\mathrm{mg} / \mathrm{ml})$ & $0.91 \pm 0.19$ & $149-416$ \\
Uric acid $(\mu \mathrm{mol} / \mathrm{l})$ & $388.02 \pm 77.37$ & $54-133$ \\
Creatinine $(\mu \mathrm{mol} / \mathrm{l})$ & $83.94 \pm 55.89$ & $2.0-7.1$ \\
Urea $(\mathrm{mmol} / \mathrm{l})$ & $5.23 \pm 1.36$ & $4-10$ \\
tPSA $(\mathrm{ng} / \mathrm{ml})$ & $1.15 \pm 1.88$ & \\
$<4$ & $245,654(96.99)$ & \\
$4-10$ & $6,468(2.55)$ & \\
$>10$ & $1,159(0.46)$ & \\
Ethnicity & & \\
Han & $255,914(98.75)$ & \\
Zhuang & $180(0.07)$ & \\
Hui & $611(0.24)$ & \\
Miao & $203(0.08)$ & \\
Tujia & $502(0.19)$ & \\
Mongolian & $139(0.05)$ & \\
Tibetan & $495(0.19)$ & \\
Yi & $152(0.06)$ & \\
Manchu & $460(0.18)$ & \\
Qiang & $225(0.09)$ & \\
Other & $265(0.10)$ & \\
\hline
\end{tabular}

Values are expressed as the mean \pm standard deviation or $n(\%)$. tPSA, total prostate-specific antigen.

tPSA levels; however, the effect size was small except in the Mongolian and Yi ethnicities. Uric acid is an antioxidant and exhibits a strong protective effect against cancer by exerting a role in the suppression of lipid peroxidation and free oxygen radical formation $(22,23)$. These properties were supported by a study in which the control group (tPSA, $0.91 \pm 0.6 \mathrm{ng} / \mathrm{ml}$ ) had significantly higher uric acid levels compared with the PCa group (tPSA, 6.87 $\pm 7.27 \mathrm{ng} / \mathrm{ml}$ ) (15). In accordance with this, the present study verified a negative correlation between tPSA levels and uric acid levels in a large population. To date, only few studies have explored the correlation of urea levels with tPSA levels and PCa (15). The present study demonstrated only a slightly positive correlation between urea levels and tPSA levels, and further high-quality studies are required for clarification in the future.

The strength of the present study was the large population, as well as the differentiation between various Chinese minority ethnicities. the present study demonstrated only a slight correlation between tPSA levels and cystatin $\mathrm{C}$, creatinine, uric acid and urea levels compared with the studies mentioned above. Thus, a clinically significant notion deduced from the present results is that patients who are only examined for those four parameters in different departments and exhibit abnormal cystatin $\mathrm{C}$ values should also be assessed for their PSA levels and risk of PCa. In particular, when creatinine, urea or cystatin $\mathrm{C}$ levels are increased in Mongolian patients, a PSA test should be considered. To the best of our knowledge, the present study was the first to investigate the correlation between tPSA levels

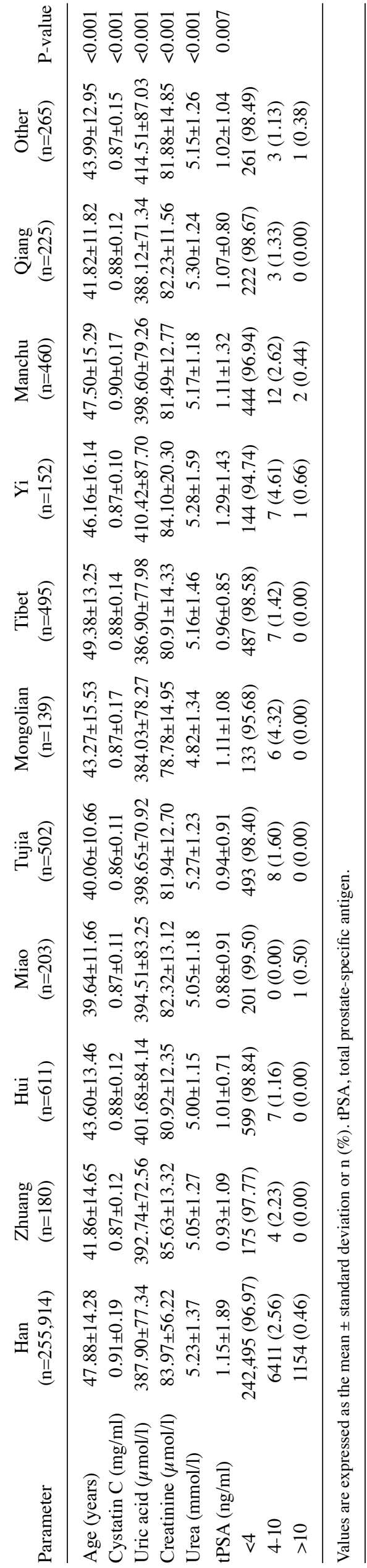



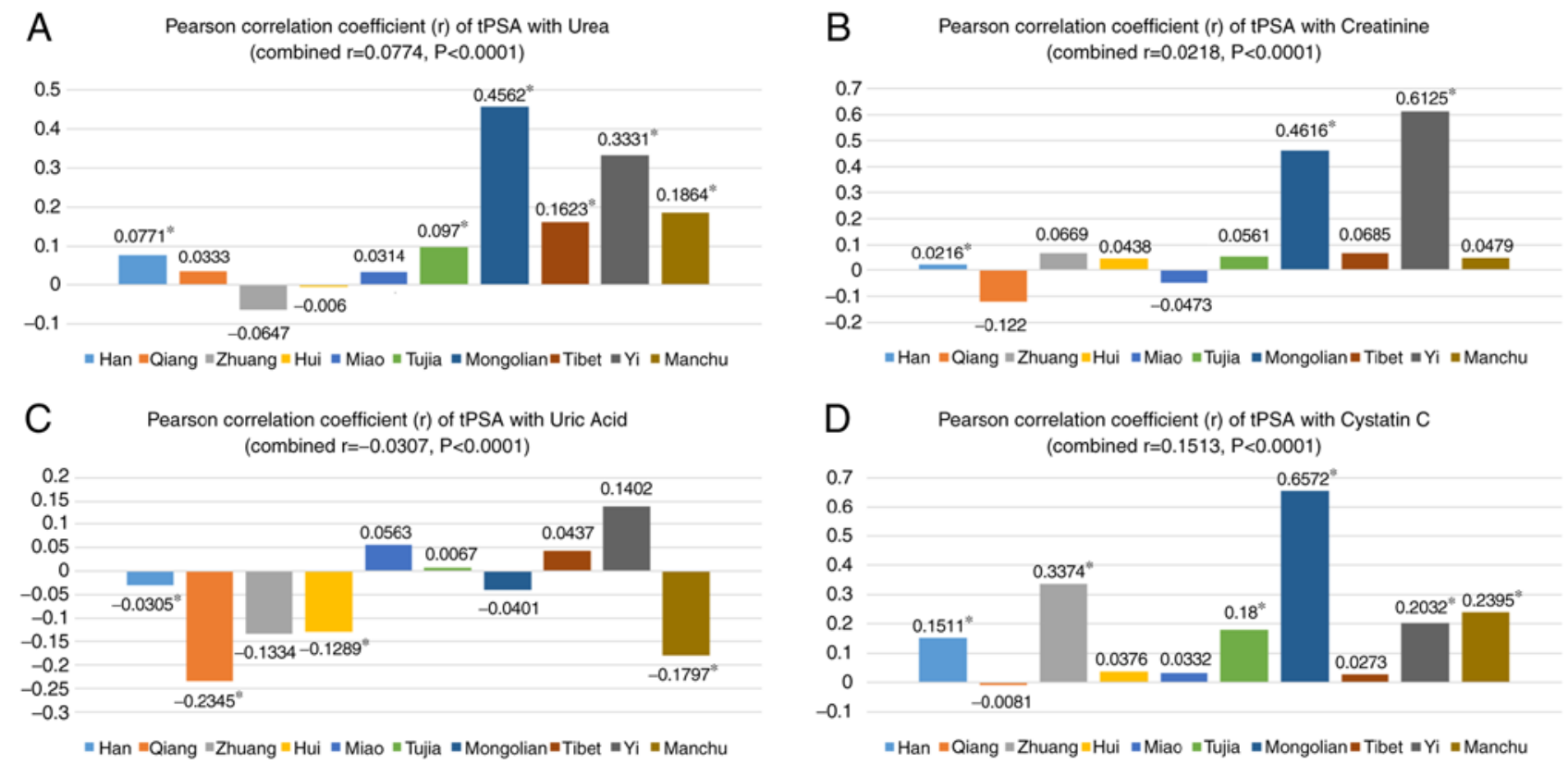

Figure 1. Pearson correlations of tPSA with urea, creatinine, uric acid and cystatin C among different ethnicities. The Y-axis indicates the Pearson correlation coefficient. (A) Pearson correlation of tPSA with urea. The correlation was slightly positive among Tibetan, Yi and Manchu ethnicities and moderately positive among Mongolians. (B) Pearson correlation of tPSA with creatinine. The correlation was moderately positive for Mongolian and Yi ethnicities. (C) Pearson correlation of tPSA with uric acid. The correlation was slightly negative among Qiang, Hui and Manchu ethnicities. (D) Pearson correlation of tPSA with cystatin C. The combined correlation was slightly positive; the correlation was also slightly positive among the Han, Zhuang, Tujia, Yi and Manchu ethnicities and moderately positive among the Mongolian ethnicity. ${ }^{*} \mathrm{P}<0.05$ regarding the correlation of two parameters in one population. tPSA, total prostate-specific antigen.

and these four parameters among different Chinese ethnicities using population-based data.

The limitations of the present study should be considered when interpreting the results. First, as a key parameter of renal function, the estimated glomerular filtration rate was not included in the analysis. Furthermore, the diagnosis of PCa was not followed up, limiting further analysis of the correlation between those parameters and $\mathrm{PCa}$. In addition, as the Han ethnicity accounted for $98.75 \%$ of the entire population of the present study, the number of patients of other minority ethnic groups was far less than that of the Han ethnicity, which may have led to potential bias. Given that the present study included 10 different ethnicities, it was not possible to further assess the data using measures such as propensity score matching for subsequent analysis. In addition, because the present study was a population-based real-world study with the goal of demonstrating differences between ethnicities, it is necessary to present the exact number of patients with different ethnicities. Furthermore, only a limited number of baseline characteristics was recorded and analyzed. Other potential confounding factors, including the age and presence of other diseases at the time of physical examination, may have also impacted the present results. Last but not the least, the present study lacked any follow-up and future studies with more complete baseline information and follow-up of $\mathrm{PCa}$ occurrence are required.

In conclusion, the present study used data from a large population to reveal a generally significant but slightly positive correlation between tPSA and cystatin C levels among the 10 most common ethnicities in China. Subgroup analyses indicated that the tPSA levels were moderately positively correlated with the creatinine level in the Mongolian and
Yi ethnicities and that the cystatin $\mathrm{C}$ level was positively correlated in the Mongolian ethnicity. The present results indicated that patients with abnormal values for these parameters should be further assessed to determine their PSA levels and subsequent risk for PCa. Future studies are certainly required to confirm and expand the present results.

\section{Acknowledgements}

Not applicable.

\section{Funding}

This program was supported by the National Key R\&D Program of China (grant nos. 2017YFC0907504 and 2017YFC0907501) and Programs from the Science and Technology Department of Sichuan Province (grant no. 2019JDR0144).

\section{Availability of data and materials}

The datasets used and/or analyzed during the current study are available from the corresponding author on reasonable request.

\section{Authors' contributions}

RL collected and analyzed data, drafted the manuscript. TB and HY conducted the statistical analysis and revised the manuscript. HT and TJ conceived the project and revised the manuscript. All authors read and approved the final manuscript and agreed to be accountable for all aspects of the work. 


\section{Ethics approval and consent to participate}

The Ethics Committee of West China Hospital (Chengdu, China) approved the present study. There was no requirement for informed consent from the patients due to the retrospective nature of the study.

\section{Patient consent for publication}

Not applicable.

\section{Competing interests}

The authors declare that they have no competing interests.

\section{References}

1. Bray F, Ferlay J, Soerjomataram I, Siegel RL, Torre LA and Jemal A: Global cancer statistics 2018: GLOBOCAN estimates of incidence and mortality worldwide for 36 cancers in 185 countries. CA Cancer J Clin 68: 394-424, 2018.

2. Aus G, Damber JE, Khatami A, Lilja H, Stranne J and Hugosson J: Individualized screening interval for prostate cancer based on prostate-specific antigen level: Results of a prospective, randomized, population-based study. Arch Intern Med 165: 1857-1861, 2005.

3. Thompson IM, Pauler DK, Goodman PJ, Tangen CM, Lucia MS, Parnes HL, Minasian LM, Ford LG, Lippman SM, Crawford ED, et al: Prevalence of prostate cancer among men with a prostate-specific antigen level $<$ or $=4.0 \mathrm{ng}$ per milliliter. N Engl J Med 350: 2239-2246, 2004.

4. Schroder FH, van der Cruijsen-Koeter I, de Koning HJ, Vis AN, Hoedemaeker RF and Kranse R: Prostate cancer detection at low prostate specific antigen. J Urol 163: 806-812, 2000.

5. Antenor JA, Han M, Roehl KA, Nadler RB and Catalona WJ: Relationship between initial prostate specific antigen level and subsequent prostate cancer detection in a longitudinal screening study. J Urol 172: 90-93, 2004.

6. Botchorishvili G, Matikainen MP and Lilja H: Early prostate-specific antigen changes and the diagnosis and prognosis of prostate cancer. Curr Opin Urol 19: 221-226, 2009.

7. Schröder FH, Hugosson J, Carlsson S, Tammela T, Määttänen L, Auvinen A, Kwiatkowski M, Recker F and Roobol MJ: Screening for prostate cancer decreases the risk of developing metastatic disease: Findings from the European Randomized Study of Screening for Prostate Cancer (ERSPC). Eur Urol 62: 745-752, 2012.

8. Heidenreich A, Abrahamsson PA, Artibani W, Catto J, Montorsi F, Van Poppel H, Wirth M and Mottet N; European Association of Urology: Early detection of prostate cancer: European Association of Urology recommendation. Eur Urol 64: 347-354, 2013.

9. Kilmer G, Roberts H, Hughes E, Li Y, Valluru B, Fan A, Giles W, Mokdad A and Jiles R; Centers for Disease Control and Prevention (CDC): Surveillance of certain health behaviors and conditions among states and selected local areas-Behavioral Risk Factor Surveillance System (BRFSS), United States, 2006. MMWR Surveill Summ 57: 1-188, 2008.
10. Nagamani SC and Erez A: A metabolic link between the urea cycle and cancer cell proliferation. Mol Cell Oncol 3: e1127314, 2016.

11. Keshet R, Szlosarek P, Carracedo A and Erez A: Rewiring urea cycle metabolism in cancer to support anabolism. Nat Rev Cancer 18: 634-645, 2018.

12. Tan P, Shi M, Chen J, Xu H, Xie N, Xu H, Jiang Y, Ai JZ, Liu LR, Yang L and Wei Q: The preoperative serum cystatin-C as an independent prognostic factor for survival in upper tract urothelial carcinoma. Asian J Androl 21: 163-169, 2019.

13. Evans TRJ, Kudo M, Finn RS, Han KH, Cheng AL, Ikeda M, Kraljevic S, Ren M, Dutcus CE, Piscaglia F and Sung MW: Urine protein:creatinine ratio vs. 24-hour urine protein for proteinuria management: Analysis from the phase 3 REFLECT study of lenvatinib vs. sorafenib in hepatocellular carcinoma. Br J Cancer 121: 218-221, 2019.

14. Selcukbiricik F, Kanbay M, Solak Y, Bilici A, Kanitez M, Balik E and Mandel NM: Serum uric acid as a surrogate marker of favorable response to bevacizumab treatment in patients with metastatic colon cancer. Clin Transl Oncol 18: 1082-1087, 2016.

15. Benli E, Cirakoglu A, Ayyildiz SN and Yüce A: Comparison of serum uric acid levels between prostate cancer patients and a control group. Cent European J Urol 71: 242-247, 2018.

16. Hashmi A, Cahill GL, Zaldana M, Davis G, Cronin BJ, Brandel MG, Beletsky A, Taj R, Buckstaff TM, Vinocur D, et al: Can head circumference be used as a proxy for intracranial volume in patients with craniosynostosis? Ann Plast Surg 82 (5S Suppl 4): S295-S300, 2019.

17. Ebert E, Werle B, Jülke B, Kopitar-Jerala N, Kos J, Lah T, Abrahamson M, Spiess E and Ebert W: Expression of cysteine protease inhibitors stefin $\mathrm{A}$, stefin $\mathrm{B}$, and cystatin $\mathrm{C}$ in human lung tumor tissue. Adv Exp Med Biol 421: 259-265, 1997.

18. Friedrich B, Jung K, Lein M, Türk I, Rudolph B, Hampel G, Schnorr D and Loening SA: Cathepsins B, H, L and cysteine protease inhibitors in malignant prostate cell lines, primary cultured prostatic cells and prostatic tissue. Eur J Cancer 35: 138-144, 1999.

19. Wegiel B, Jiborn T, Abrahamson M, Helczynski L, Otterbein L, Persson JL and Bjartell A: Cystatin C is downregulated in prostate cancer and modulates invasion of prostate cancer cells via MAPK/Erk and androgen receptor pathways. PLoS One 4: e7953, 2009.

20. Weinstein SJ, Mackrain K, Stolzenberg-Solomon RZ, Selhub J, Virtamo J and Albanes D: Serum creatinine and prostate cancer risk in a prospective study. Cancer Epidemiol Biomarkers Prev 18: 2643-2649, 2009.

21. Wang M, Zou L, Liang J, Wang X, Zhang D, Fang Y, Zhang J, $\mathrm{Xiao} \mathrm{F}$ and Liu M: The urinary sarcosine/creatinine ratio is a potential diagnostic and prognostic marker in prostate cancer. Med Sci Monit 24: 3034-3041, 2018.

22. Kolonel LN, Yoshizawa C, Nomura AM and Stemmermann GN: Relationship of serum uric acid to cancer occurrence in a prospective male cohort. Cancer Epidemiol Biomarkers Prev 3: 225-228, 1994

23. Ames BN, Cathcart R, Schwiers E and Hochstein P: Uric acid provides an antioxidant defense in humans against oxidant- and radical-caused aging and cancer: A hypothesis. Proc Natl Acad Sci USA 78: 6858-6862, 1981. 